\title{
Single diagonal precast prestressed concrete bracing for strengthening existing concrete frames
}

\author{
Fariborz Nateghi Alahi ${ }^{1}$ (i) $\cdot$ Mohsen Vatandoost ${ }^{2}$
}

Received: 30 November 2017 / Accepted: 19 September 2018 / Published online: 3 October 2018

(c) The Author(s) 2018

\begin{abstract}
A new method for seismic retrofitting concrete structures is proposed using an $\mathrm{X}$-shaped precast prestressed concrete brace. This PPC brace is made of four precast concrete parts and middle section that are assembled and added to the existing frame. This method has the following benefits: there is no need to work with wet concrete in site or anchor and bolt to the existing frame, which may lead to a fast and economic retrofitting method. The X-shaped concrete brace was made in half size and its efficiency is confirmed. That X-shaped precast prestressed concrete brace was simulated and evaluated by computerized analysis using ABAQUS FEA modeling by authors of this paper earlier, and the proposed brace showed proper results in reducing lateral drifts and that method is considered as a proper method for seismic retrofitting. Here we are suggesting a different setup for the brace, which may lead to faster and more economic strengthening of RC structures. The new setup is omitting the middle section and suggests the brace to be used as a single diagonal. A model of this proposed method is simulated in ABAQUS FEA and the results show it is proper in reducing lateral displacements.
\end{abstract}

Keywords Seismic retrofitting $\cdot$ Seismic strength $\cdot$ Concrete braces $\cdot$ Single diagonal braces $\cdot$ Precast concrete $\cdot$ Prestressed concrete $\cdot$ Reinforced concrete structure

\section{Novelty and research method}

The aim of this research is to evaluate a modified proposed setup using precast prestressed concrete (PPC) braces, suggested by the authors of this paper. VATANABE and partners formerly proposed a method for seismic retrofitting of the reinforced concrete structures (Watanabe et al. 2004; Watanabe and Kono 2006) which has many advantages, such as there is no need to work with wet concrete in site and no need to anchor or bolt to the existing frame which may lead to a fast and economic retrofitting method. The X-shaped concrete brace, which was modeled in half size by VATAN$\mathrm{ABE}$ and some subsequent researcher, was evaluated and its efficiency is confirmed (Nateghi et al. 2018a, b; Vatandoost 2010; Kono and Katayama 2009; Watanabe et al. 2002).

Fariborz Nateghi Alahi

nateghi@iiees.ac.ir

Mohsen Vatandoost

mohsen.vatandoost@gmail.com

Department of Structure, IIEES, Tehran, Iran

2 Islamic Azad University, Tehran, Iran
The authors of this paper studied the X-shaped concrete brace by simulated analysis model of bracing system using ABAQUS FEA modeling (Hibbett et al. 1998; Bathe 2006), by comparing the existing frame and retrofitted frame; the results showed that this system is proper in reducing lateral displacement (Nateghi 2018; Nateghi and Vatandoost 2018b). Here we are suggesting a different setup for the brace which may lead to faster and more economical strengthening of RC structures. The new setup omits the middle section and suggests the brace to be used as a single diagonal. A model of this proposed method is simulated in ABAQUS FEA and the results are analyzed.

\section{Introduction: precast prestressed concrete bracing system}

Structure frames with brace rather than other structures such as moment frames or shear walls are an effective and valuable way for retrofitting against lateral loads (earthquake and wind) because incline members act as truss which bears tensile and compression tensions. The behavior of reinforced concrete braced frame is studied and confirmed (Desai et al. 


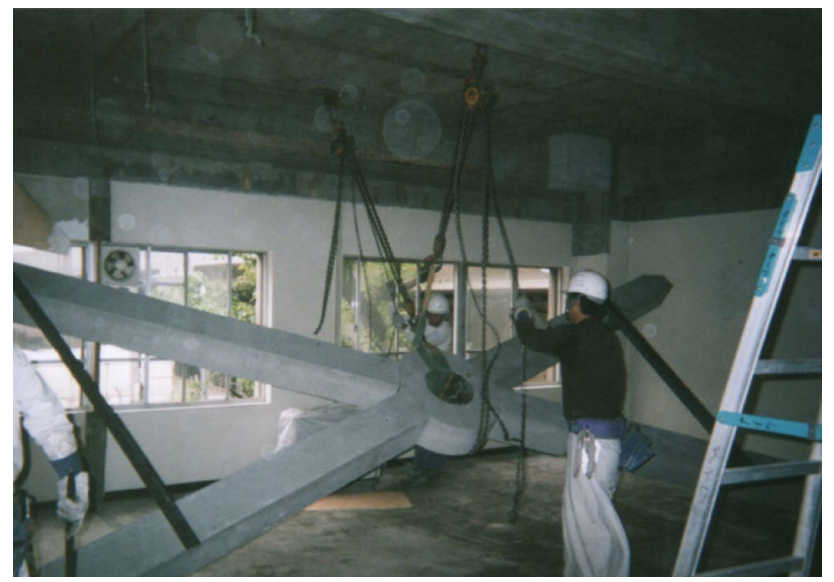

Fig. 1 The erecting of brace (Watanabe et al. 2004)

1988; Iskhakov 2001; Khaloo and Mohseni 2008; Xu and Niu 2003; Rodriguez and Park 1991; Ohishi et al. 1988; Yazgan and Nishiyama 2008). The X-shaped precast prestressed concrete brace which is proposed by VATANABE and partners (Watanabe et al. 2004; Watanabe and Kono 2006) is made of four precast concrete legs, those parts and the middle section are assembled in the site and the prestress force is applied to the assembled system among the end legs of the braces. Then the brace is fitted to the existing frame as shown in Fig. 1 and the end joint between brace and frame is filled by an none-shrinkage high-strength mortar in the other joints an L-shape plate is used to hinder compression failure between frame and brace. After hardening of mortar the prestress force is freed and brace is extended and placed to the frame. Figure 2 indicates the final placement of the brace; as the figure shows, there is still enough opening for day light and ventilation. When braced frame is under

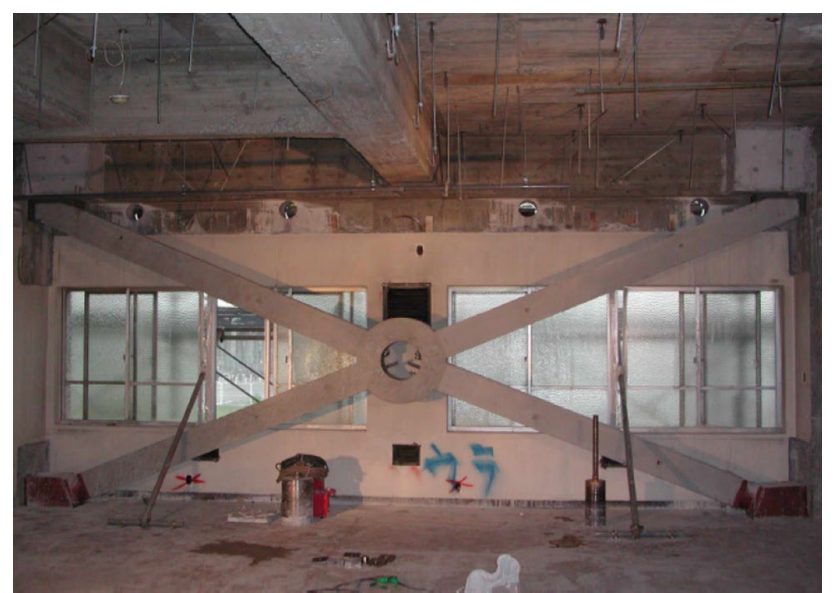

Fig. 2 The X-shaped brace is assembled and is placed in the existing frame (there is enough opening for day light and ventilation) (Watanabe et al. 2004, 2006)

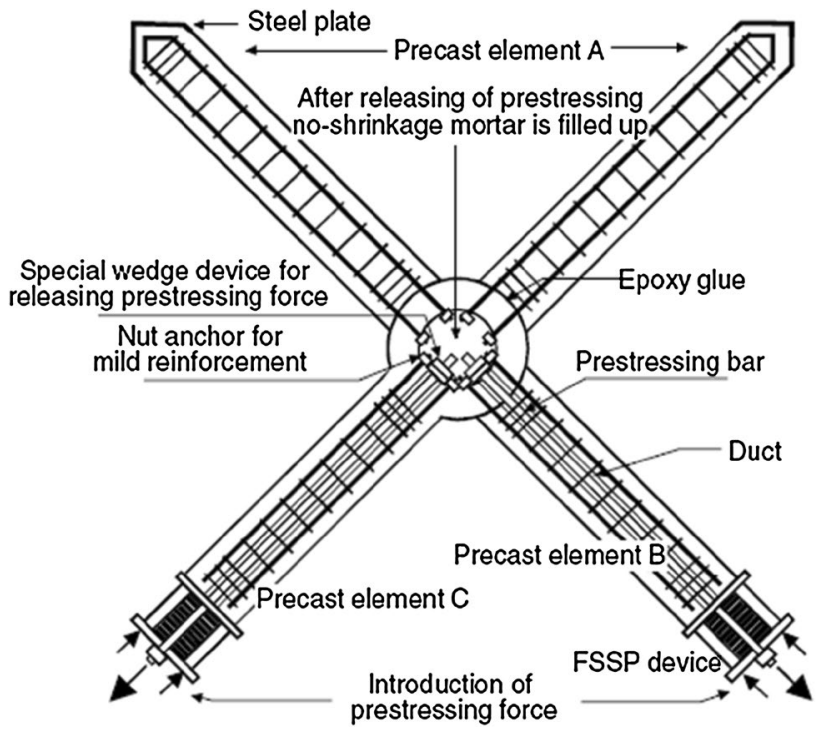

Fig. 3 Precast legs are assembled and the prestressing force is applied (Watanabe et al. 2004)

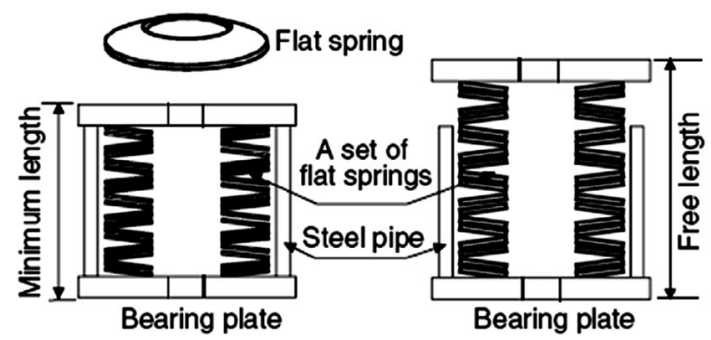

Fig. 4 FSSP device (flat springs steel pipe) (Watanabe et al. 2004, 2006)

compression force due to lateral force (earthquake) only one leg of X-shaped brace is effective in compression; without using prestress force, tensile leg of brace would detach from the frame, because concrete tensile strength is inconsiderable. To prevent this, at the end of the leg two of brace a special device which is called FSSP (Flat Springs Steel Pipe) is used (Figs. 2, 3). The FSSP is used in both ends of X-shaped brace as is shown in Fig. 3. This device is providing a limited compression force among the brace even if the brace is due to tensile force while the FSSP device is applying compression force. Therefore, both legs remain in compression during the response to earthquake and lateral loads.

VATANABE and partners modeled two frames by a scale of 0.5 to real frame, The results of the experimental work showed that the proposed $\mathrm{X}$-shaped brace is proper in reducing lateral displacements (Watanabe et al. 2004; Watanabe and Kono 2006).

Then that existing frame which was needed to be seismically retrofitted according to Japanese code by 
VATANABE assessment (Watanabe et al. 2004) was simulated by Hibbett et al. (1998); Bathe (2006) by authors of this paper (for more details, please see the reference Nateghi and Vatandoost (2018a, b). The experimental and analytical results of braced frame were compared. Assessments by comparing model no. 1 and model no. 2 to the existing frame indicated that the $\mathrm{X}$-shaped bracing system is proper in reducing lateral displacements and when the ratio of compression strength of brace to frame is more than two (Figs. 4, 5) the bracing system is much more effective (Nateghi and Vatandoost 2018a). In Fig. 6, lateral force-displacement of existing frame and braced frame models no. 1 and no. 2 is compared. It concluded that applying precast prestressed concrete braces would adds extra stiffness to the frame. All three models (primary frame and braced frame models no. 1 and no. 2) were loaded due to failure; Fig. 5 shows that model no. 1 did not show much stiffness and it yielded due to brace buckling, Braced frame model No. 2 which the brace compression strength was four times greater than model No. 1 added proper and effective stiffness to the frame and lateral displacements of frame were reduced from $325 \mathrm{~mm}$ in primary frame to $17 \mathrm{~mm}$ in model No. 2 (Nateghi and Vatandoost 2018a). Figure 5 also indicates the failure mode of models due to lateral force in analysis. The failure mode of primary frame was due to shear in columns and model no. 1 failure mode was buckling of brace and model no. 2 failed by shear at the end of the beam-column joint. By considering failure mode, practical and analytical practices were greatly in conformity which indicates the accuracy of simulating.

For more information and details about PPCB, the reader is referred to Watanabe et al. (2004), Watanabe and Kono (2006), Nateghi and Vatandoost (2018a, b) and Vatandoost (2010).

\section{Introduction proposal, modified precast prestressed concrete bracing system}

\section{Single diagonal brace by omitting middle section- model no. 1}

In proposed modified model, we suggest to omit the middle section and a single diagonal brace in two in a row existing frame (Fig. 7) instead of X-shaped brace. The most signifying property of the proposed modified system is faster and easier installation of the system which may lead to more economic seismic retrofitting method. By elimination of the middle section, there is no need of leg one and leg two bars to intersect in the middle section.

Similar to the previous model, at the end of the leg two of the brace there is a FSSP device to prevent the

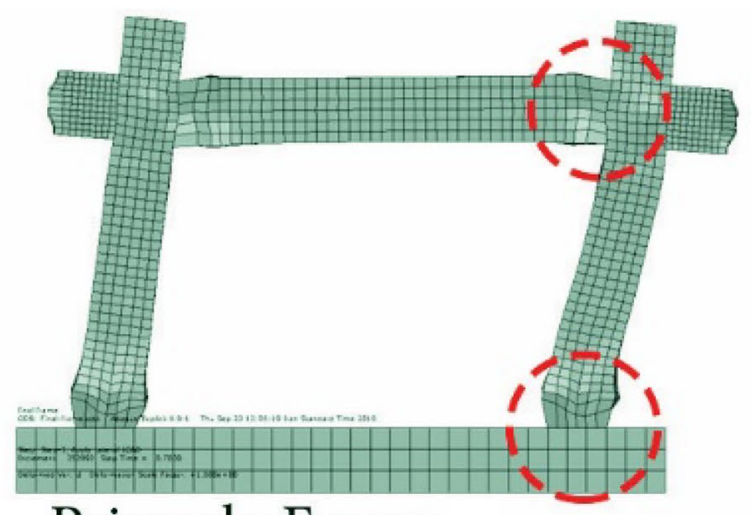

\section{Primarly Frame}
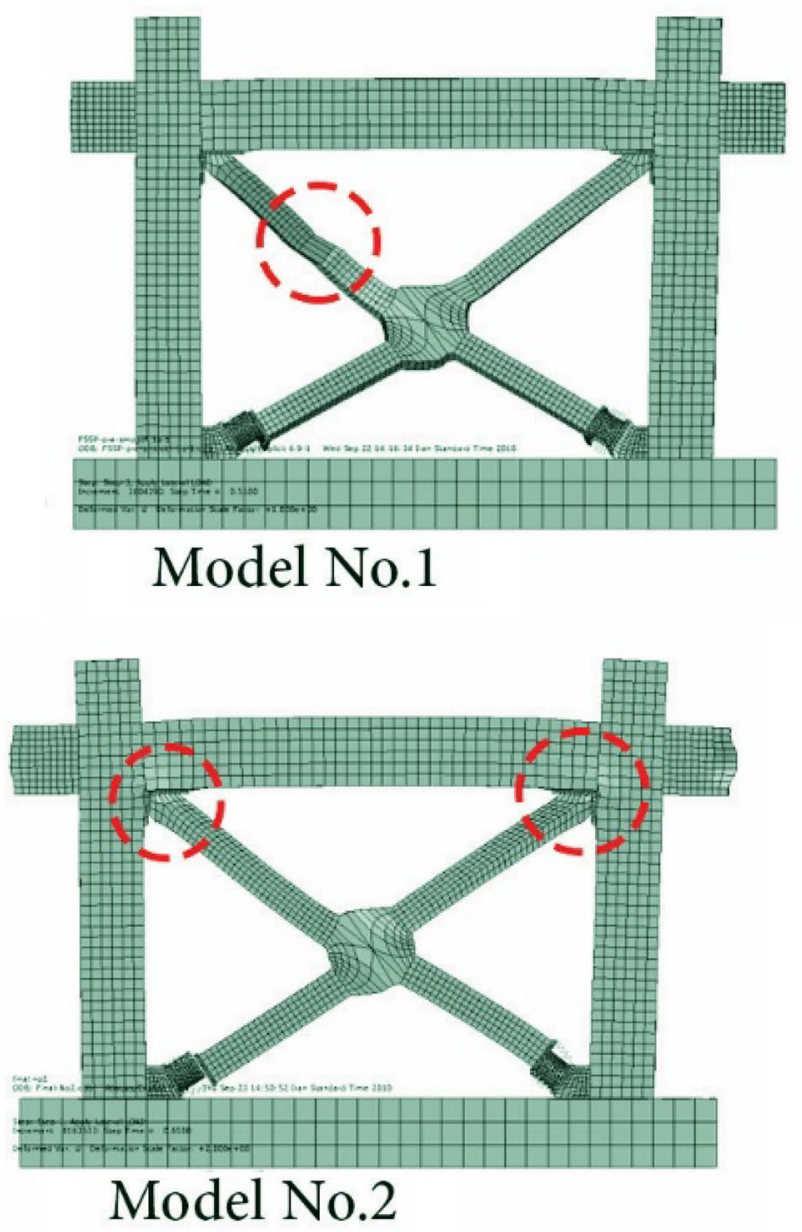

Fig. 5 All models and their failure modes. a Primary frame, $\mathbf{b}$ braced frame model no. 1 and $\mathbf{c}$ braced frame model no. 2 (Nateghi and Vatandoost 2018a)

separation of the brace from frame in tensile. The proposed modified bracing system consists of two single diagonal precast concrete braces in two in a row (adjacent) frames. 
Fig. 6 Lateral force-displacement primary frame and braced frame models no. 1 and no. 2 (Vatandoost 2010)

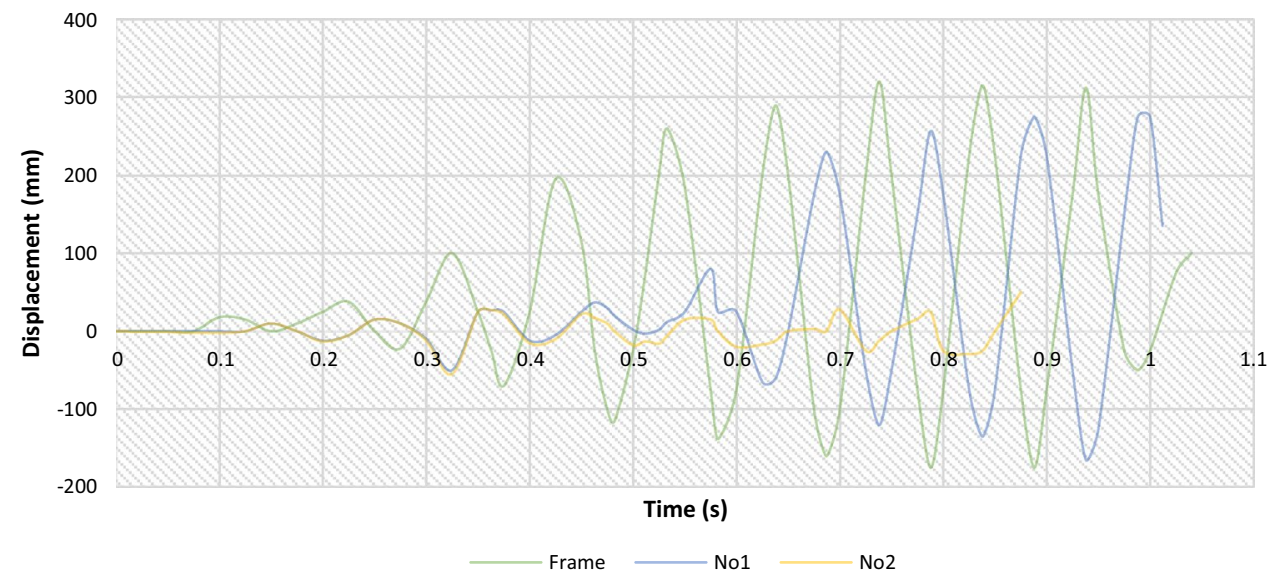

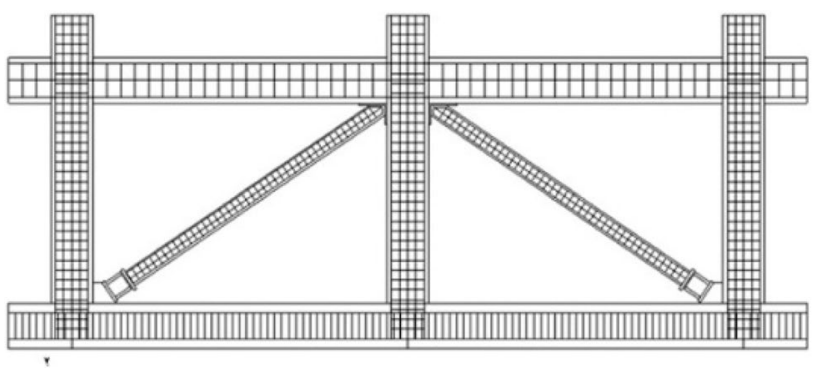

Fig. 7 Modified and proposed single diagonal bracing system by omitting the middle section

Due to lateral cyclic forces, each time one of the single diagonal braces is in compression and works properly and because concrete tensile strength is not considerable, one of those diagonals by elongation of the FSSP device is in tensile and does not participate in lateral force bearing. For seismic evaluation and assessment of the proposed bracing system a model is simulated in ABAQUS FEA. The properties of primary frame which need to be seismically retrofitted is a reinforced concrete frame of a building which needs to be seismically retrofitted according to the Japanese code (Watanabe et al. 2004; Watanabe and Kono 2006; Nateghi and Vatandoost 2018a). Brace dimension is similar to model no. 1 and is $100 \times 120 \mathrm{~mm}$. (The selection of brace dimension and also properties of brace was due to the previous $\mathrm{X}$-shaped brace used for retrofitting the existing frame, and here we did not change the properties, we just changed the configuration to compare diagonal brace with $\mathrm{X}$-shaped brace.) The main bars of brace are four D6 and shear bars are D4 with $50 \mathrm{~mm}$ space along the brace. Figure 8 indicates the bar layout in simulated model. (Figs. 9, 10, 11, 12, 13, 14, 15, 16, 17, 18, 19, 20, 21).

Lateral loading procedure was used as it was discussed in reference Nateghi and Vatandoost (2018a, b) which was applied to braced system model no. 1 in reference Nateghi

Fig. 8 Bar layout in the simulated model (modified proposed bracing system

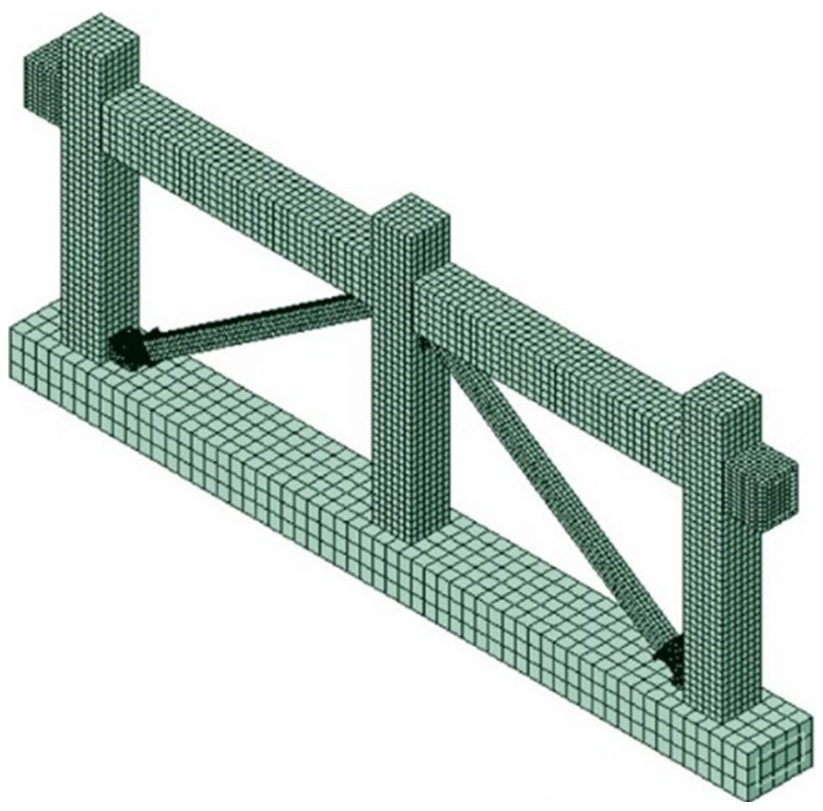

Fig. 9 3D model of braced frame in ABAQUS

and Vatandoost (2018a) (a very short summary is that solving the protocol step by step simulated the loading correctly, such protocol consists of applying gravity and prestress 


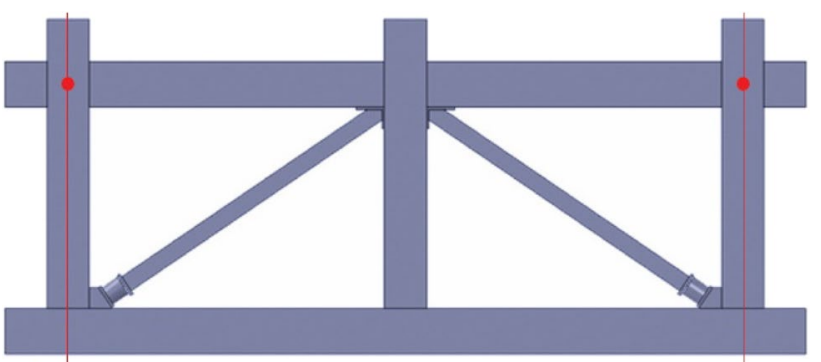

Fig. 10 Position of strain gages in simulated model for measuring lateral displacements

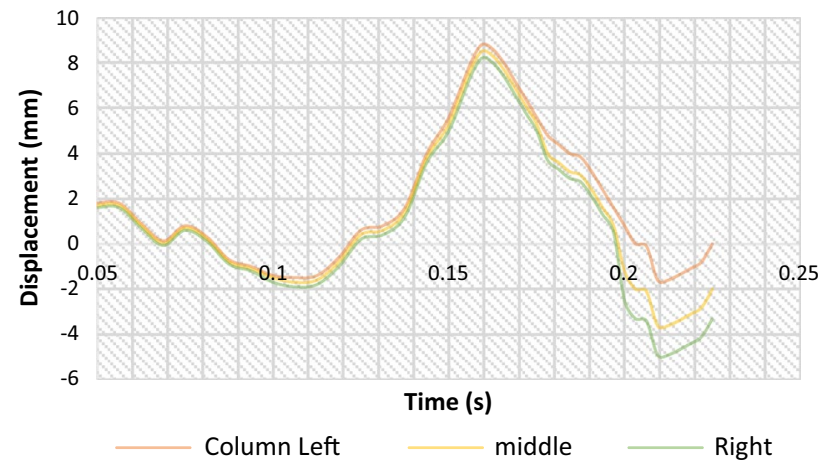

Fig. 11 Displacement-time chart for points indicated in single diagonal brace model

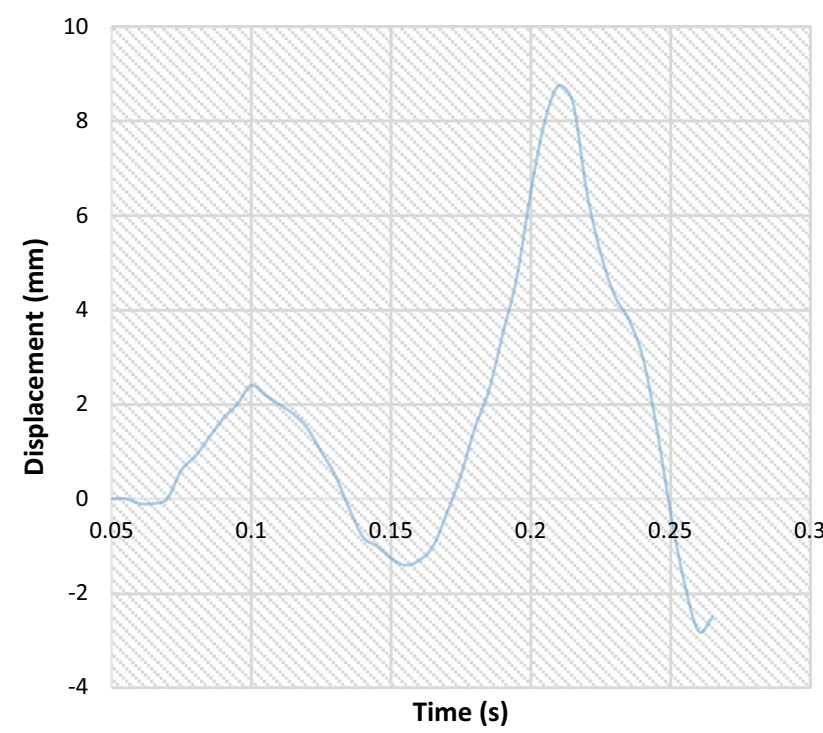

Average Displacement-Time Chart for points indicated in single diagonal brace model

Fig. 12 Average displacement-time chart for points indicated in single diagonal brace model

force and then lateral force in separate consequent steps. The results-outputs - which are provided in each step are the input of the next step.).

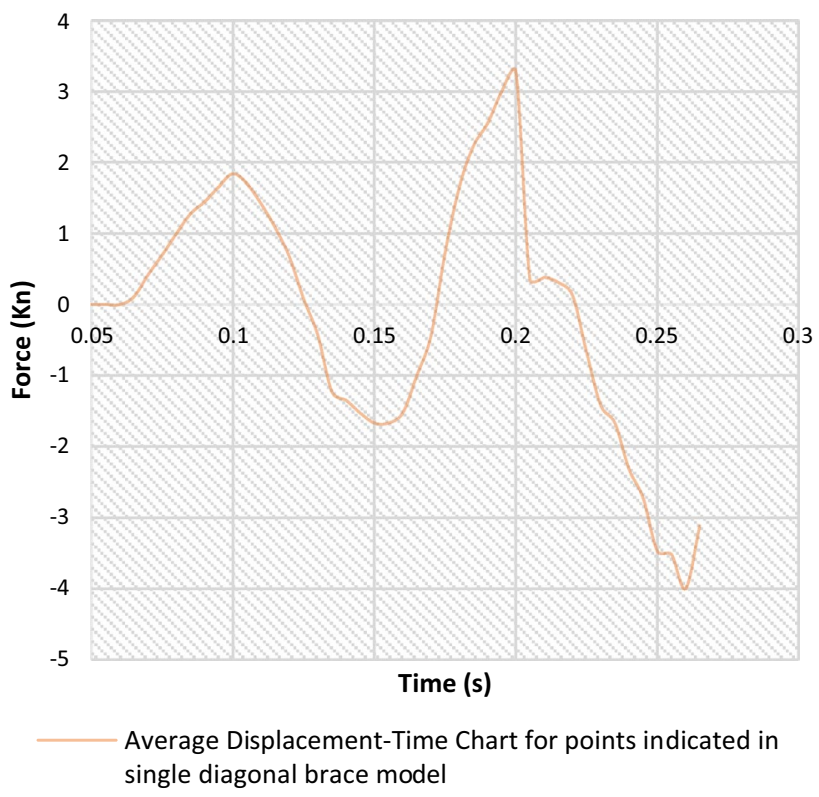

Fig. 13 Force (kn)-time(s) chart for single diagonal brace model

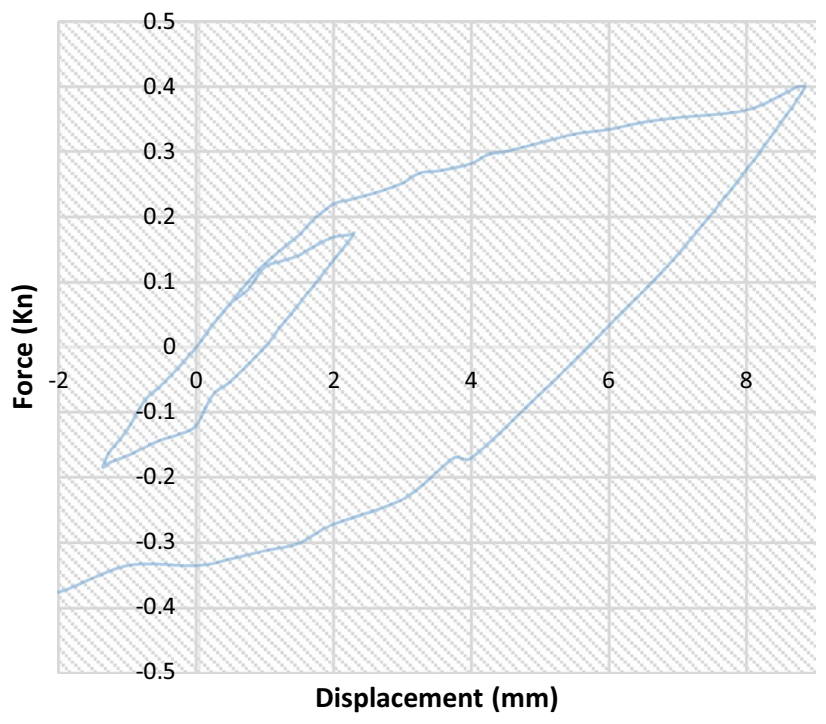

Fig. 14 Force-displacement for single diagonal brace model

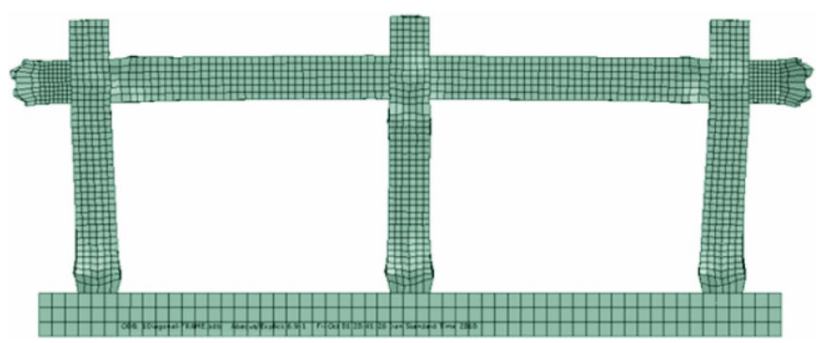

Fig. 15 Failure mode of primary frame (which has been assessed to need a seismic retrofitting) 
Fig. 16 Failure mode of diagonal braced system—buckling of brace
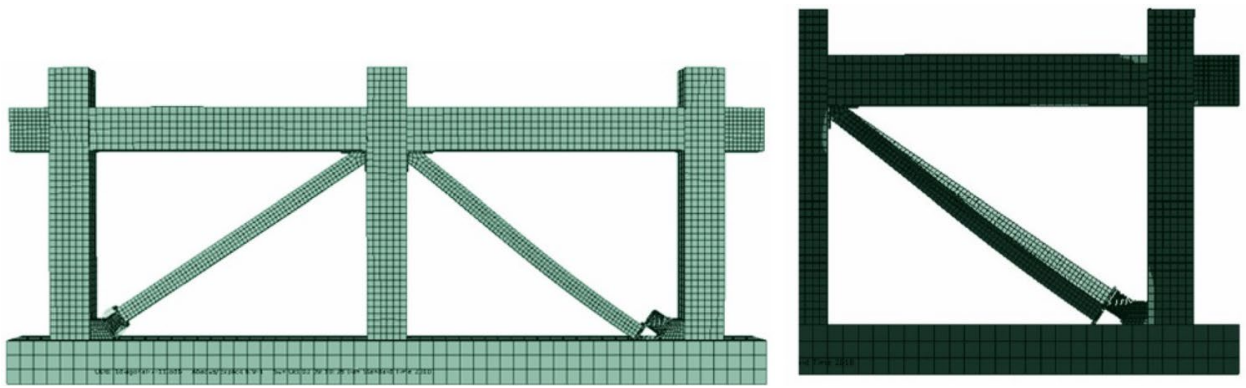

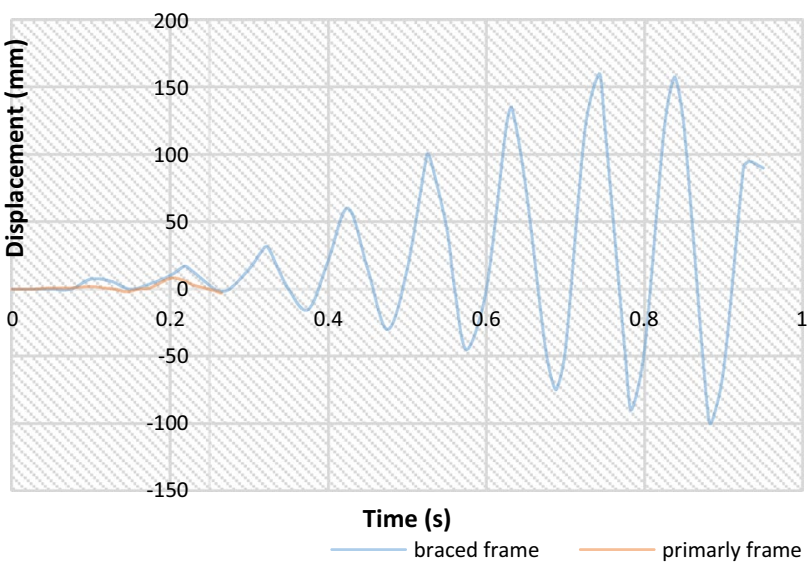

Fig. 17 Comparison between primary frame and braced frame

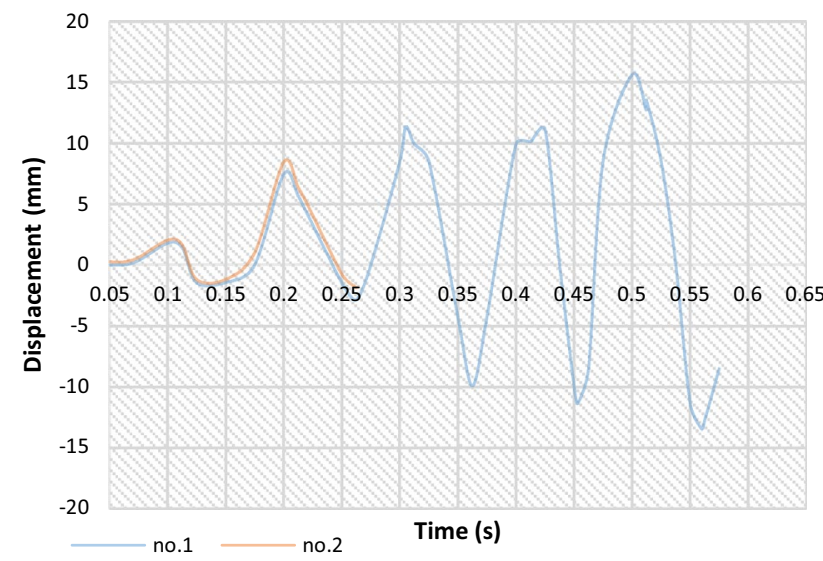

Fig. 18 Comparison of lateral displacement between diagonal brace model no. 1 and model no. 2

In this model, gravity loads of the above story are ignored to simplify the model and reduce very long solvation time demanded by the software. Diagonal brace was prestressed by applying 40-kn tensile force. After installation of brace in frame and using none-shrinkage high-strength mortar at the end of the FSSP after hardening of mortar the prestress force is released which leads to elongation of diagonal brace and the brace is fitted in the frame. Then lateral force is applied to

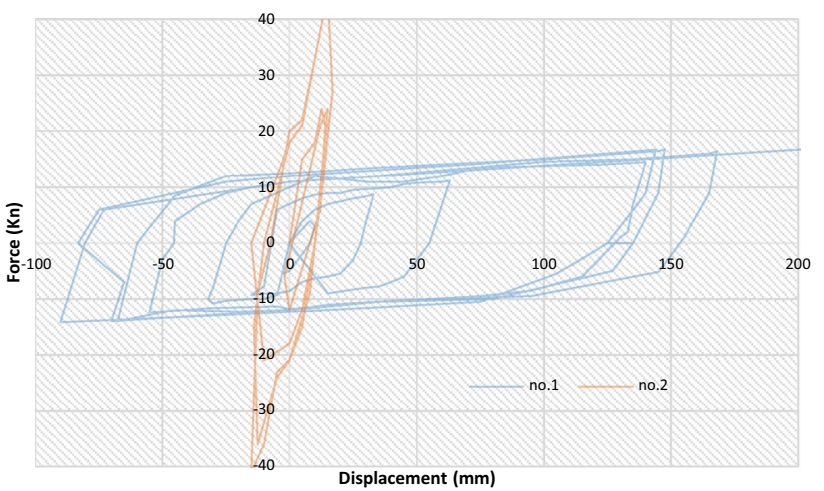

Fig. 19 Force-displacement chart provided for model no. 1 and model no. 2

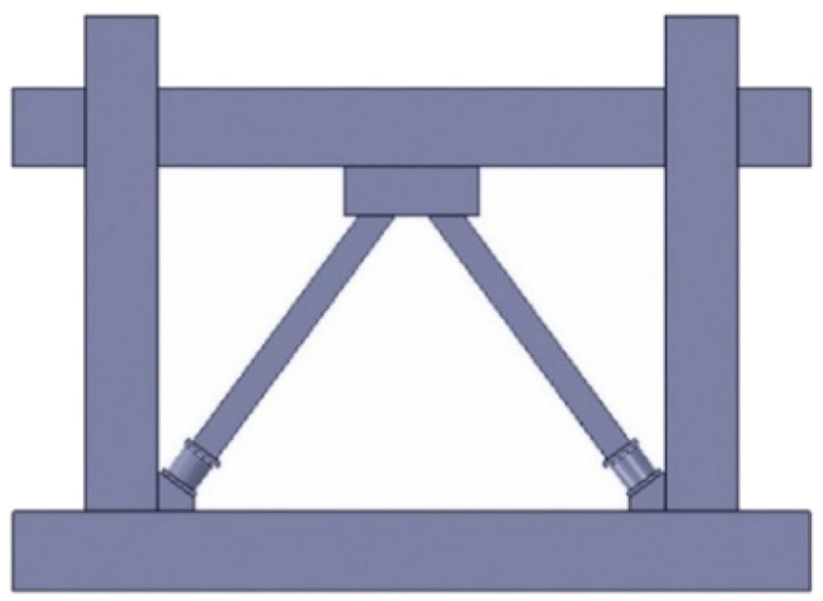

Fig. 20 Proposed V-shaped bracing system

the model. The model failure mode was brace buckling as it is shown in Fig. 16.

The lateral displacement is computed by averaging displacements provided from two oints which are indicated in Fig. 12. (These points are those at which strain gages are presumed to be installed.) 


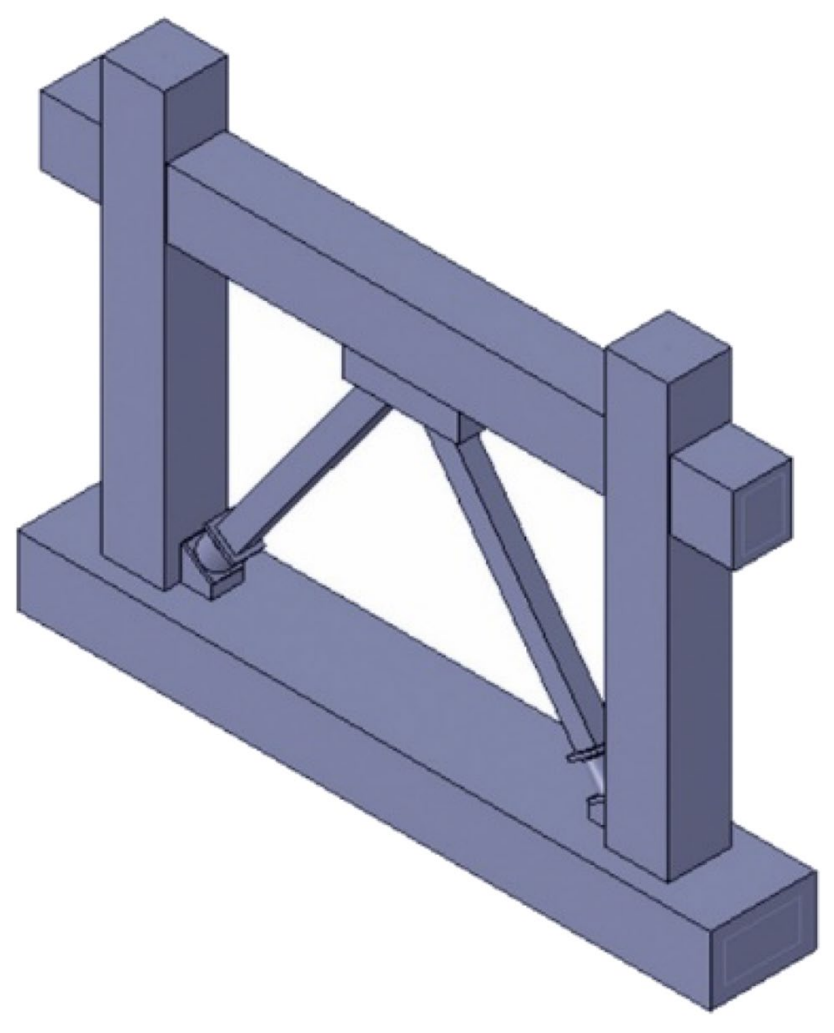

Fig. 21 3D view of the proposed V-shaped bracing system

\section{Comparison of seismic parameters of primary frame and single diagonal braced frame}

The failure mode of primary frame was shear failure of columns which is provided in Fig. 15. In addition, failure mode of single diagonal braced frame was buckling of brace. Figure 16 shows the failure mode of brace frame.

\section{Single diagonal brace by omitting middle section- model no. 2}

The chart provided in Fig. 17 shows lateral displacement-time relation in primary and braced frame. This chart shows although the braced has reduced lateral displacements, the brace has failed by buckling very soon and could not resist enough against lateral force (Figs. 14, 16). In addition, because the slender ratio of single diagonal brace has increased, using thicker section or less slender ratio than brace model no. 1 is required. Therefore, in model no. 2 , the compression strength of brace is increased from 27.9 to 82.7 MPa (three times growth). Braced system is subjected to lateral force; results are provided in Figs. 17 and 18.

Model no. 2 failure mode was similar to model no. 1buckling of brace — but it carried proper lateral load and was effective in lateral displacement reduction as is shown in chart of Fig. 19. It can be concluded from charts in Figs. 17 and 18 that single brace model no. 2 which is similar to $\mathrm{X}$-shaped brace model no. 1 reduces lateral displacements effectively but the slender ratio of brace should be reduced by choosing a thicker brace or using brace with more compression strength to hinder (postpone) the buckling of brace. Design of proper section is beyond the realm of current paper and it is suggested for future researches.

\section{The proposal of V-shaped bracing system}

The proposed brace consists of two precast concrete diagonal legs that are connected in the middle of the beam in a $\mathrm{V}$-shaped setup. This proposed V-shaped seams have the benefits of the two last proposed systems, $\mathrm{X}$-shaped and single diagonal. In this proposed brace, the middle section is omitted like single diagonal brace which may lead to faster and more economical seismic method and unlike the single diagonal the length of each leg is reduced (in comparison to single diagonal brace) and the result is that slender ratio of brace is reduced which leads to more lateral load bearing before buckling of brace. But the challenge is connecting the brace to the beam properly and dealing with shear force applied by brace to beam. Design of proper section is beyond the realm of the current paper and it is suggested for future researches.

\section{Summary}

1. VATANABE and partners formerly have proposed an X-shaped precast prestressed concrete braces for seismic retrofitting of RC structures. That method was assessed as a reasonable method by practical and analytical works (Watanabe et al. 2004; Watanabe and Kono 2006).

2. A model of precast prestressed concrete braces has been simulated formerly by the authors of this paper using ABAQUS FEA (Nateghi and Vatandoost 2018a, b) and the $\mathrm{X}$-shaped brace was found to be proper in reducing lateral displacement. Here we have proposed a new setup for braces by omitting the middle section of the X-shaped brace. The modified proposed bracing system consists of single diagonal braces in two adjacent frames. This single diagonal proposed brace has not only all of the great properties of the $\mathrm{X}$-shaped brace but also omitting the middle section may lead to a faster and more economical seismic retrofitting method.

3. The most signifying properties of the proposed braced are that unlike the X-shape there is no need for four legs of the brace to intersect at the middle and there 
is no need for grout and glume legs of the brace to be together which may lead to faster and more easier and more economical way of strengthening seismically.

4. A model of the proposed single diagonal brace is simulated by ABAQUS FEA by comparison of displacement-time charts of primary frame and braced frame model no. 1 that are shown in Fig. 17. It can be concluded that single diagonal braces are effective in reducing lateral displacements.

5. In model no. 1, although the brace has reduced lateral displacements, the brace has failed by buckling very soon and could not resist enough against lateral force. In addition, because the slender ratio of single diagonal brace has increased, using thicker section or less slender ratio than brace model no. 1 is needed. Therefore, in model no. 2, the compression strength of the brace is increased from 27.9 to $82.7 \mathrm{MPa}$ (three times growth) and the braced system is subjected to lateral force (Results are provided in Figs. 18 and 19).

6. Failure mode of single diagonal brace no. 1 (Fig. 16) indicates that by increasing the length of the brace in comparison to $\mathrm{X}$-shaped brace, buckling of brace is more probable, and using thicker section or less slender ratio than brace model no. 1 is needed.

7. In model no. 2 in which compression strength of brace is increased from 27.9 to $82.7 \mathrm{MPa}$ (three times growth to model no. 1), the buckling of brace was the reason of failure but model no. 2 endured more lateral forces (Fig. 19) and lateral displacement decreased from $25 \mathrm{~mm}$ in model no. 1 to $10 \mathrm{~mm}$ in model no. 2 .

8. In X-shaped brace, the leg one of the brace which was in tensile has helped the hindrance (postponed) of brace buckling but in single diagonal brace thicker or greater slender ratio is needed to postpone buckling of brace. Results showed that the brace has not buckled properly in reducing lateral displacement.

9. For this single diagonal bracing method to be efficient, the properties and brace section to primary frame should be chosen so that the brace would not buckle before beam or column of frame and could dissipate most of the energy of lateral forces and reduce drifts and improve seismic strength of the existing frame.

10. Another proposed setup of precast prestressed concrete brace is V shaped; this proposed brace consists of two precast concrete diagonal legs that are connected in the middle of the beam (Figs. 20, 21). This proposed $\mathrm{V}$ shape seems to have the benefits of both X shape and single diagonal, the middle section is omitted like the single diagonal brace which may lead to faster and more economical seismic method and unlike the single diagonal the length of each leg is reduced that hinders (postponed) buckling of brace. The challenge is proper connection of the brace to beam and dealing with shear force applied by brace to beam.

11. We offer the following guidelines in future researches: studying the use of FRP (fiber-reinforced polymers) for strengthening the frame and postponing concrete braces buckling; using fiber concrete for creating braces; study of use of high-strength concrete in braces; dynamic and non-linear assessments of precast prestressed concrete braces and finding more proper and optimizing properties of L-shaped end plate and none-shrinkage mortar.

Suggestion for future researchers:

- Use of CFRP and GFRP (Oda et al. 2008) to strength the concrete brace and enhance its performance.

- Using fibrous concrete (Amit 2017) for concrete braces.

Acknowledgements The authors thank the kind support provided by the International Institute of Earthquake Engineering and Seismology of Iran-IIEES — for helping in completing this project.

Open Access This article is distributed under the terms of the Creative Commons Attribution 4.0 International License (http://creativeco mmons.org/licenses/by/4.0/), which permits unrestricted use, distribution, and reproduction in any medium, provided you give appropriate credit to the original author(s) and the source, provide a link to the Creative Commons license, and indicate if changes were made.

\section{References}

Amit S (2017) Study on retrofitting of reinforced concrete beams using fibrous concrete. J Struct Transp Stud 2(1):1-8

Bathe KJ (2006) Finite element procedures. Klaus-Jurgen Bathe

Desai JP, Jain AK, Arya AS (1988) Seismic response of R.C. braced frames. Comput Str 29(4):557-568

Hibbett, Karlsson, Sorensen (1998) ABAQUS/standard: user's manual, vol 1. Hibbett, Karlsson \& Sorensen

Iskhakov I (2001) Self variable stiffness system for optimal seismic response of RC frame with concrete braces. Trans Built Environ 57:201-210

Khaloo AR, Mohseni MM (2008) Nonlinear seismic behavior of RC frames with RC braces. Asian J Civil Eng (Build Hous) 9(6):577-592

Kono S, Katayama T (2009) Seismic retrofit of reinforced concrete building structures with prestressed braces. J Adv Concr Technol 7(3):337-345

Nateghi FA, Vatandoost M (2018a) Seismic retrofitting RC structures with precast prestressed concrete braces-ABAQUS FEA modeling. Int J Eng 31(3):394-404

Nateghi AF, Vatandoost M (2018b) Seismic retrofitting RC structures with precast prestressed concrete braces. J Int Inst Earthq Eng Seismol I. R. Iran 20:1145-115

Oda M, Kono S, Watanabe F (2008) Seismic retrofit of RC buildings with prestressed precast CFT and FRC braces. In: The 14th world conference on earthquake engineering, Beijing, China, 12-17 October 2008 
Ohishi H, Takahashi M, Yamazaki Y (1988) A seismic strengthening design and practice of an existing reinforced concrete school building in Shizuoka city. In: Proceedings of the ninth world conference on earthquake engineering, pp 415-420

Rodriguez M, Park R (1991) Repair and strengthening of reinforced concrete buildings for seismic resistance. Earthq Spectra 7(3):439-459

Vatandoost M (2010) Seismic Retrofitting RC Structures With precast prestressed concrete braced, MSc thesis, Azad University, Takestan branch, september 2010. (under super vision of Professor F.Nateghi-A)

Watanabe F (2002) Development of the "self-installing PCa brace method for seismic reinforcement of building walls

Watanabe F, Kono S (2006) Retrofiting RC structures with precast prestressed concrete braces. In: Proceeding of the 8th US national conference on earthquake engineering, April 18-22, 2006, San Francisco, California, USA, paper No. 635
Watanabe F, Miyazaki S, Tani M, Kono S (2004) Seismic strengthening using precast prestressed concrete braces. In: 13th world conference on earthquake engineering, Vancouver, B.C., Canada, 1-6 August, 2004, paper No. 3406

Xu S, Niu D (2003) Seismic behavior of reinforced concrete braced frame. ACI Struct J 100(1):120-125

Yazgan AU, Nishiyama M (2008) Seismic performance evaluation of existing RC structures according to the Japanese standard and retrofitting with prestressed precast CFT and FRC braces. Bull Int Inst Seismol Earthq Eng 42:115-120

Publisher's Note Springer Nature remains neutral with regard to jurisdictional claims in published maps and institutional affiliations. 